\title{
GLACIER VOLUME CHANGE ESTIMATION USING TIME SERIES OF IMPROVED ASTER DEMS
}

\author{
Luc Girod $^{\mathrm{a} *}$, Christopher Nuth ${ }^{\mathrm{a}}$ and Andreas Kääb ${ }^{\mathrm{a}}$ \\ a Department of Geosciences, University of Oslo, Postboks 1047 Blindern, 0316 Oslo, Norway \\ (luc.girod, christopher.nuth, andreas.kaeaeb)@geo.uio.no
}

Commission VIII, WG VIII/6

KEY WORDS: ASTER, Jitter, DEM generation, RPC, Glacier

\begin{abstract}
:
Volume change data is critical to the understanding of glacier response to climate change. The Advanced Spaceborne Thermal Emission and Reflection Radiometer (ASTER) system embarked on the Terra (EOS AM-1) satellite has been a unique source of systematic stereoscopic images covering the whole globe at $15 \mathrm{~m}$ resolution and a consistent quality for over 15 years. While satellite stereo sensors with significantly improved radiometric and spatial resolution are available to date, the potential of ASTER data lies in its long consistent time series that is unrivaled, though not fully exploited for change analysis due to lack of data accuracy and precision. Here, we developed an improved method for ASTER DEM generation and implemented it in the open source photogrammetric library and software suite MicMac. The method relies on the computation of a rational polynomial coefficients (RPC) model and the detection and correction of cross-track sensor jitter in order to compute DEMs. ASTER data are strongly affected by attitude jitter, mainly of approximately $4 \mathrm{~km}$ and $30 \mathrm{~km}$ wavelength, and improving the generation of ASTER DEMs requires removal of this effect. Our sensor modeling does not require ground control points and allows thus potentially for the automatic processing of large data volumes.

As a proof of concept, we chose a set of glaciers with reference DEMs available to assess the quality of our measurements. We use time series of ASTER scenes from which we extracted DEMs with a ground sampling distance of $15 \mathrm{~m}$. Our method directly measures and accounts for the cross-track component of jitter so that the resulting DEMs are not contaminated by this process. Since the along-track component of jitter has the same direction as the stereo parallaxes, the two cannot be separated and the elevations extracted are thus contaminated by along-track jitter. Initial tests reveal no clear relation between the cross-track and along-track components so that the latter seems not to be easily modeled analytically from the first one. We thus remove the remaining along-track jitter effects in the DEMs statistically through temporal DEM stacks to finally compute the glacier volume changes over time. Our method yields cleaner and spatially more complete elevation data, which also proved to be more in accordance to reference DEMs, compared to NASA's AST14DMO DEM standard products.

The quality of the demonstrated measurements promises to further unlock the underused potential of ASTER DEMs for glacier volume change time series on a global scale. The data produced by our method will help to better understand the response of glaciers to climate change and their influence on runoff and sea level.
\end{abstract}

\section{INTRODUCTION}

\subsection{Motivation}

Glaciers and their changes over time are key elements of the Earth's water cycle influencing global sea level and, in many mountain regions, local water availability for household, agricultural and hydropower use. Glacier can also pose significant natural hazards, for instance through ice avalanching and glacier lake outbursts. Volume changes of glaciers reflect glacier mass changes and are thus critical to the understanding of glacier response to climate change. The Advanced Spaceborne Thermal Emission and Reflection Radiometer (ASTER) system on the Terra (EOS AM-1) spacecraft has been a unique source of systematic stereoscopic images covering the whole globe at consistent characteristics for over 15 years. While satellite stereo data with significantly improved radiometric and spatial resolution are available to date, the potential of ASTER data lies in its long time series that is unrivaled, though by far not fully exploited for change analysis due to lack of data accuracy and precision. Long time series are crucial for glacier change detection in order to separate short-term variations in surface mass balance from the more long-term changes driven by variations in climate and glacier dynamics (Wang and Kääb, 2015). Only recently, the ASTER data

${ }^{*}$ Corresponding author archive has been made freely available to the public which further calls for and facilitates improved and systematic exploitation of these stereo data over large regions.

\subsection{Challenges}

Even if the existence of such raw data over such a long time span is a great starting point, DEMs generated by NASA with SilcAst (SILC, 2015) (ASTER DMO products) do not provide a sufficient geometric quality for glacier volume change estimation over short periods, the expected change being significantly smaller than the accuracy of the product (a few meters against $\pm 30 \mathrm{~m}$, see Figure 1 of the DMO over a scene of fast sea ice, i.e. with expected completely flat topography). This is due in part to the unaccounted for satellite jitter inducing attitude perturbation (Kääb et al., 2013). Proposed causes for this disturbance include the mechanical cooling system, the rotation of mirrors and movements of the high gain antenna, but a source is yet to be formally identified.

Due to our goal to develop a method for automatic processing over large areas and data volumes, and the fact that glacier environments change significantly over time, it is complicated and inefficient to use ground control points (GCPs). Ground truth obtained by other methods on stable terrain suffers from lack of availability. The SRTM mission covers only $60^{\circ} \mathrm{N}$ to $56^{\circ} \mathrm{S}$, for 
instance, while the TanDEM-X iDEM is not yet complete and freely available but will have to be examined in this context.

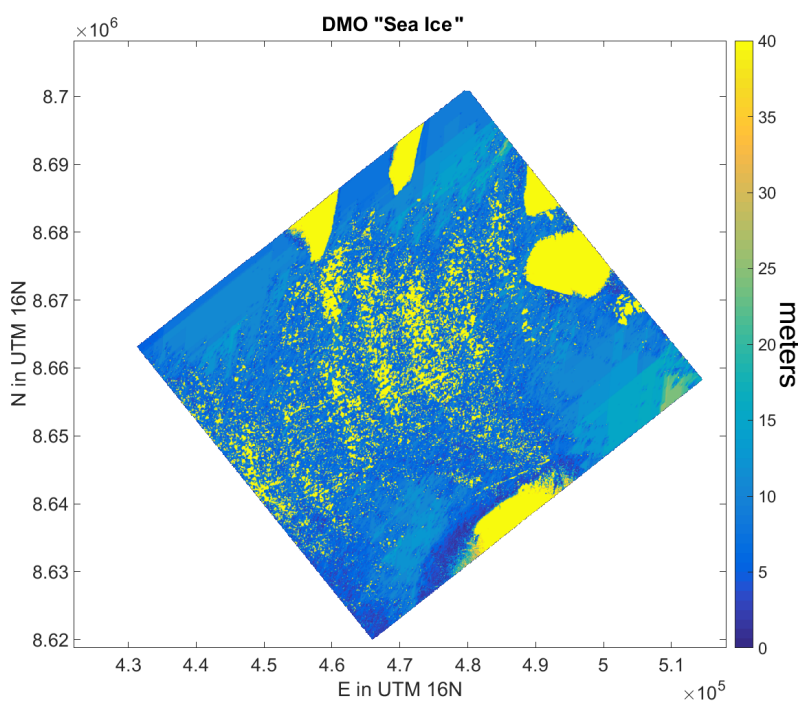

Figure 1: ASTER DMO product over a fast sea ice scene, which should be uniformly flat in the sea area, presents gaps filled with interpolated data and high noise, the data peaks at several hundred meters. The high elevation areas (yellow) at the scene margins represent islands.

In (Girod et al., 2015) and (Kääb et al., 2013), we discussed the characteristics of the ASTER jitter signal and showed that compensation methods previously proposed for other sensors or even other bands of the ASTER system were not applicable. We then demonstrated that it was possible to compute improved DEMs, being more complete and with a clean along-track signal that we supposed could be removed using a stack of DEMs and stable terrain. In this paper, we shortly explain the DEM extraction method in section 2.1 and then how we remove the remaining along-track signal in section 2.2 .

\section{MMASTER METHOD}

Our method, dubbed MMASTER to reflect the use of MicMac in this ASTER processing chain, is using for its first part the DEM generation method presented in (Girod et al., 2015) and recapped in section 2.1. The second part of the method, presented in section 2.2, allows for the generation of either DEMs if an external dataset with good data on the stable terrain is available, or elevation change values if two (or more) ASTER scenes of the same area at different points in time are available. Figure 2 summarizes the different steps of the method.

\subsection{DEM extraction}

In (Girod et al., 2015), we presented a method to extract a DEM from the VNIR images of an ASTER L1A dataset with a correction on the cross-track jitter. The method can be divided in the following steps :

- Extraction from HDF file: nadir and back-looking VNIR images, radiometric calibration tables and geolocation data (lattice points in both geocentric and image coordinates every 400 pixels along the image's Y-axis as well as the associated satellite position).

- Destriping of the images by the application of the radiometric calibration tables to the images.

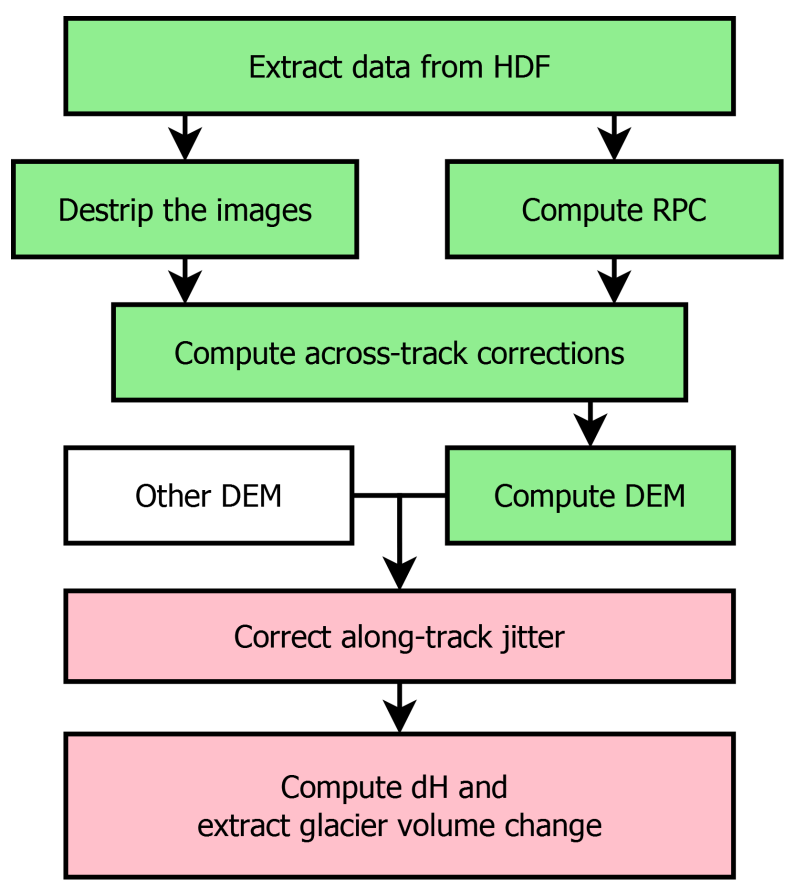

Figure 2: Diagram of the overall method. Green, as presented in (Girod et al., 2015) and recapped in section 2.1. Red, presented in this paper in section 2.2.

- Creation of grids of points for each image at regular $(500 \mathrm{~m})$ altitude intervals on the lattice points-defined lines of sights (see Figure 3).

- Fitting of RPC functions (see equations 1 to 5) on the grid using mean square minimization.

- Computing of the cross-track jitter component by estimating the distance between the point of best correlation and the epipolar line and correcting the image for it (see example of a correction grid in Figure 4).

- Computing the DEM by searching for the point of best correlation for given altitudes for all points on the $15 \mathrm{~m}$ GSD UTM coordinate grid of the imaged area.

$$
\begin{aligned}
& \text { Lon }_{\text {norm }}=\frac{P_{1}\left(\operatorname{Col}_{\text {norm }}, \text { Row }_{\text {norm }}, h_{\text {norm }}\right)}{P_{2}\left(\operatorname{Col}_{\text {norm }}, \text { Row }_{\text {norm }}, h_{\text {norm }}\right)} \\
& \text { Lat }_{\text {norm }}=\frac{P_{3}\left(\operatorname{Col}_{\text {norm }}, \text { Row }_{\text {norm }}, h_{\text {norm }}\right)}{P_{4}\left(\operatorname{Col}_{\text {norm }}, \text { Row }_{\text {norm }}, h_{\text {norm }}\right)} \\
& \text { Col }_{\text {norm }}=\frac{P_{5}\left(\text { Lon }_{\text {norm }}, \text { Lat }_{\text {norm }}, h_{\text {norm }}\right)}{P_{6}\left(\text { Lon }_{\text {norm }}, \text { Lat }_{\text {norm }}, h_{\text {norm }}\right)} \\
& \text { Row }_{\text {norm }}=\frac{P_{7}\left(\text { Lon }_{\text {norm }}, \text { Lat }_{\text {norm }}, h_{\text {norm }}\right)}{P_{8}\left(\text { Lon }_{\text {norm }}, \text { Lat }_{\text {norm }}, h_{\text {norm }}\right)}
\end{aligned}
$$

With :

$$
\begin{aligned}
P_{i}(X, Y, Z)=C_{1}+C_{2} X+C_{3} Y+C_{4} Z \\
\quad+C_{5} X Y+C_{6} X Z+C_{7} Y Z+C_{8} X^{2}+C_{9} Y^{2}+C_{10} Z^{2} \\
+C_{11} X Y Z+C_{12} X^{3}+C_{13} X Y^{2}+C_{14} X Z^{2}+C_{15} X^{2} Y \\
\quad+C_{16} Y^{3}+C_{17} Y Z^{2}+C_{18} X^{2} Z+C_{19} Y^{2} Z+C_{20} Z^{3}
\end{aligned}
$$




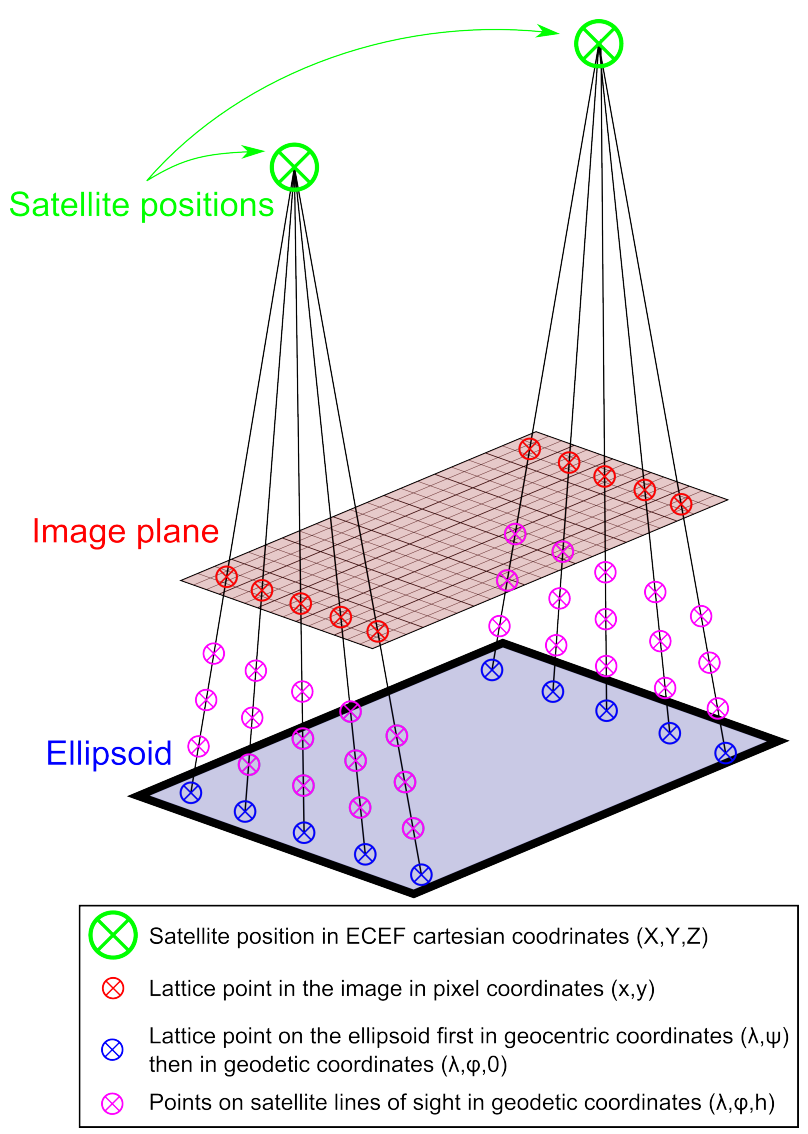

Figure 3: Satellite positions and associated lattice points in image and geographical coordinates and 3D GRID created from the lattice points.

\subsection{Along-track jitter removal}

To obtain good elevation difference data, the DEMs produced by the aforementioned method still require post-processing. Indeed, the along-track jitter component is still present in the DEMs. Because of its relatively low amplitude compared to the terrain variation in most scenes, it is not easy to detect the along-track jitter effect from the single DEM produced alone. However, we are interested in elevation change, and computing the elevation difference between two scenes largely removes the terrain signal and shows the superposition of the jitter effects from both DEMs. Actual terrain change (such as glacier elevation change, erosion, or similar processes) is also added to the signal. Figure 5 shows the difference of two DEMs after a 3D co-registration using the method described in (Nuth and Kääb, 2011).

To remove this remaining effect composed of the along-track jitters of the two ASTER DEMs, we first need to filter out the unstable terrain. This can be done by masking changing terrain manually, by using a database of glacier areas and a buffer to account for possible glacier changes over time or database inaccuracies, or by detecting glaciers using multispectral methods such as band ratios (Winsvold et al., 2016). This last method, while being automated as well as not requiring external data, works on cleanice glaciers but might fall short on debris covered glaciers and other non-glacier phenomena such as landslides . Note also, that ASTER SWIR data is not anymore available for multispectral analysis in images acquired in recent years due to sensor degradation.

When only the stable terrain is remaining, the data is fit with fifth degree polynomials in the across and along track directions for

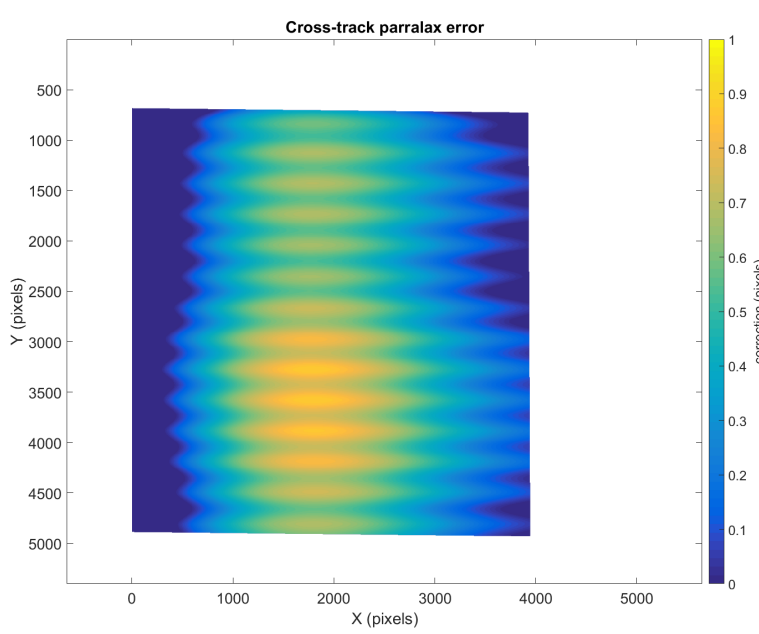

Figure 4: Measure of the combined cross-track vibration visible in the back-looking image through bi-dimensional correlation in back-looking image coordinates for the 2014 scene presented in section 3.1.

the long wavelength part $(\sim 34 \mathrm{~km})$ and by a sinusoidal signal (with known bounds on wavelength $(4.5 \pm 0.3 \mathrm{~km})$ and amplitude $(5 \pm 5 m))$ in the along-track direction for the short wavelength part. Depending on the amount and distribution of stable terrain in the scene available for fitting, the signal might be difficult to identify and therefore only partially corrected. Figure 6 shows the difference between the two study DEMs after the application of the above corrections.

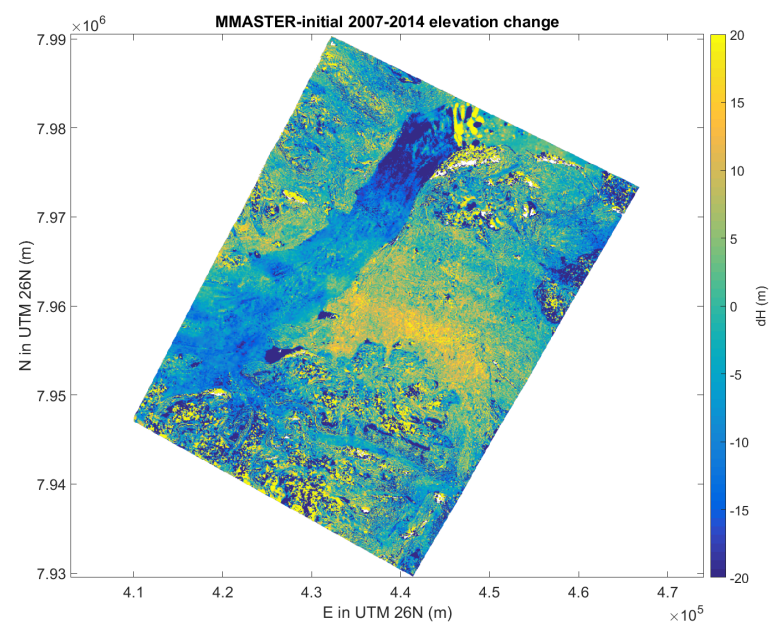

Figure 5: Example of measured elevation change from two ASTER DEMs after co-registration. For an overview of the area covered see Figure 7.

\section{GLACIER VOLUME CHANGE}

\subsection{Test scene}

For our test, we needed an area with two ASTER scenes acquired at timestamps corresponding to the acquisition of independent data. We found such an area around Daugard-Jensen glacier in eastern Greenland. It was covered by data from the SPIRIT project (SPOT 5 stereoscopic survey of Polar Ice: Reference Images and Topographies, (Korona et al., 2009)) and by ASTER in 2007 and 2014 nearly simultaneously (with respectively 4 and 10 days delays between the two systems. The references and exact dates of the acquisitions are listed in Table 1). 


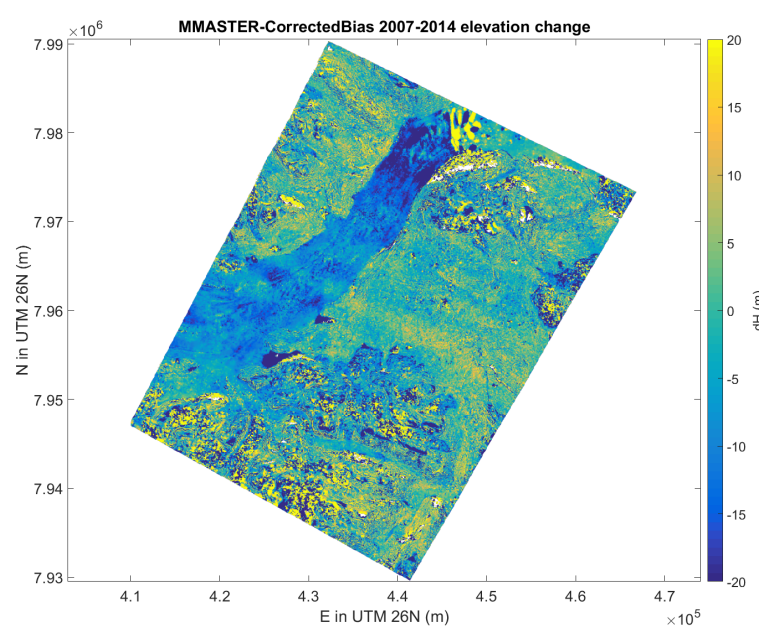

Figure 6: Example of measured elevation change after correction of the along-track jitter effect. Some jitter remains visible pointing to a still imperfect correction. For an overview of the area covered see Figure 7.

The SPIRIT project used SPOT 5 HRS stereopairs to create DEMs over selected important polar regions at 40m GSD in UTM projection. The accuracy of the DEMs produced by the SPOT 5 HRS system has been assessed at around $5 \mathrm{~m}$ (relative) and 10/15 $\mathrm{m}$ (absolute) (Baudoin et al., 2004). After co-registration of two of these products, we can therefore expect an accuracy of the elevation difference of around $7 \mathrm{~m}$.

\begin{tabular}{|l|c|}
\hline \multicolumn{2}{|c|}{ ASTER } \\
\hline Date & $2007-07-29$ \\
Reference & AST_L1A\#00307292007135727_08012007140410 \\
\hline Date & 2014-06-12 \\
Reference & AST_L1A\#00306122014141003_06132014033533 \\
\hline \hline \multicolumn{2}{|c|}{ SPIRIT } \\
\hline Date & 2007-08-02 \\
Reference & GES_08-015 \\
\hline Date & 2014-06-02 \\
Reference & GES_14-012 \\
\hline
\end{tabular}

Table 1: Dataset references.

\subsection{Data}

Elevation change grids were computed for each dataset with coregistered products (the SPIRIT scenes together on one hand see Figure 9 - and the two DMO scenes together on the other hand - see Figure 10), or, for the MMASTER product, after application of our method.

Our analysis focuses on the Daugaard-Jensen glacier part that is visible is both ASTER scenes as well as on a group of small glacier unlinked to the Greenland Ice Sheet. The approximate centerline and the selected areas are shown in Figure 7.

We first extracted the 2007-2014 elevation change values over the centerline for each dataset. The difference between the SPIRIT elevation change values (considered ground truth with a $\pm 7 \mathrm{~m}$ accuracy) and both the DMO and MMASTER values are plotted in Figure 8. The MMASTER values are in most part (89\%) inside of the $\pm 7 \mathrm{~m}$ accuracy band given by the SPIRIT data (agreement of $-2.5 \pm 4.0 \mathrm{~m}$ ) while the DMO is significantly off (agreement of $+11.2 \pm 28.2 m$, with only $24 \%$ in the accuracy band). The DMO data shows both error trends and sharp error peaks reaching over $100 \mathrm{~m}$ while the MMASTER is fairly contained. While the error trend should stem from the long-wavelength jitter effects not

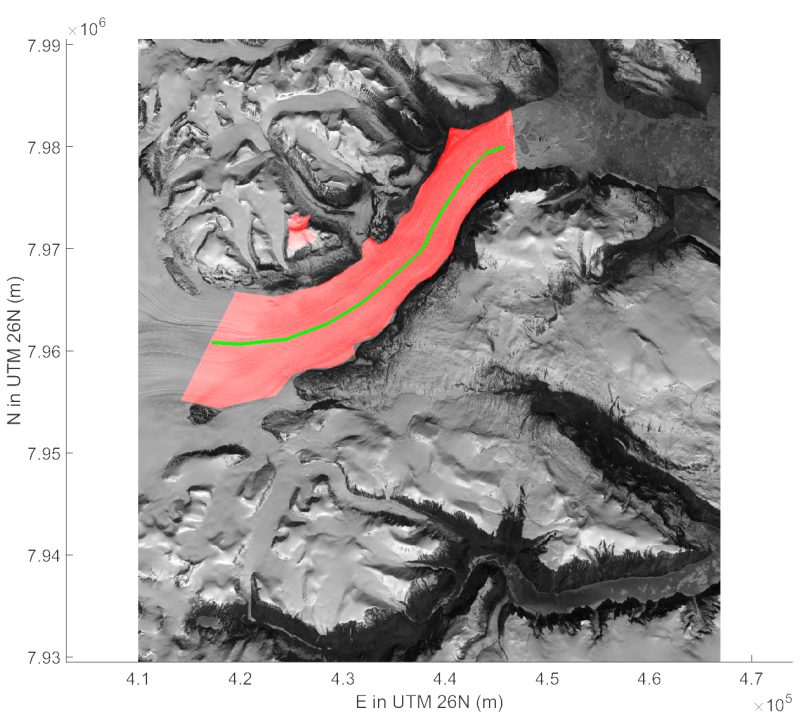

Figure 7: Centerline (green) and areas (red) considered for the quantitative analysis, overlaying the SPIRIT 2014 orthoimage.

accounted for in the DMO in contrast to the MMASTER DEM, we assume that the sharp error peaks could in parts be due to the cross-track jitter not accounted for in the DMO processes. Corresponding points in the ASTER stereo data can have a cross-track offset from the (approx. along-track) epipolar line that is too large to match between them when using a directional (i.e. along-track) correlation algorithm with insufficient cross-track margin.

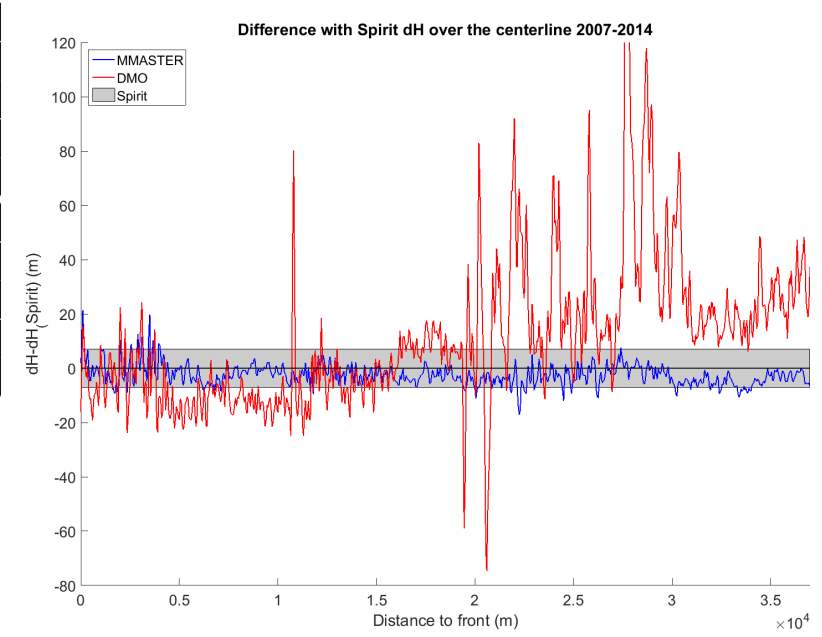

Figure 8: Difference along the centerline (shown in Figure 7) between the 2007-2014 elevation change from the SPIRIT data (set as reference data and thus zero; error bar shown in grey; see Figure 9) and the elevation change from our product (MMASTER, see Figure 6) and the DMO product (see Figure 10).

We then compared the volume change computed for the both the selected area of the main glacier and a group of small glaciers using each dataset(see Table 2). For the Daugaard-Jensen outlet glacier section, it appears that while the MMASTER and the SPIRIT data are relatively in accordance with a $36 \%$ overestimation of the volume loss from the MMASTER data compared to the SPIRIT data, the DMO data shows significantly different values with a $-130 \%$ underestimate of the volume loss hence concluding that the glacier is experiencing volume gain. For the small group of glaciers, the MMASTER and the SPIRIT data are showing very similar numbers ( $4 \%$ underestimate for the MMASTER) while the DMO data shows a $-154 \%$ underestimate again wrongly concluding that the group of glaciers is gaining volume. 
On both cases, the MMASTER outperforms the DMO in terms of accuracy with reference to the SPIRIT DEM differences.

\begin{tabular}{|l|c|c|}
\hline Dataset & dV Daugaard-Jensen & dV Group \\
\hline \hline DMO & $+0.629 \mathrm{~km}^{3}$ & $+0.014 \mathrm{~km}^{3}$ \\
\hline MMASTER & $-2.805 \mathrm{~km}^{3}$ & $-0.025 \mathrm{~km}^{3}$ \\
\hline SPIRIT & $-2.055 \mathrm{~km}^{3}$ & $-0.026 \mathrm{~km}^{3}$ \\
\hline
\end{tabular}

Table 2: Volume change in glacier tongue according to each dataset.

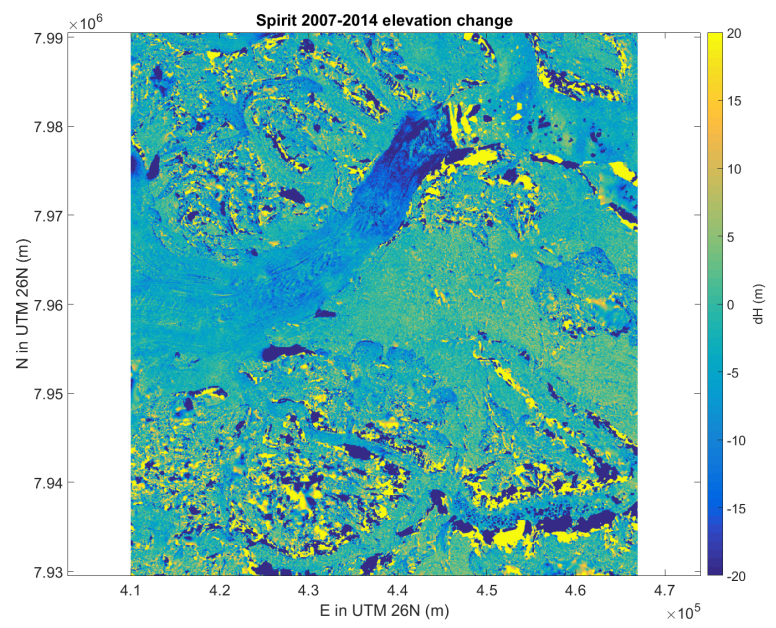

Figure 9: Measured elevation change from co-registered 2007 and 2014 SPIRIT DEMs.

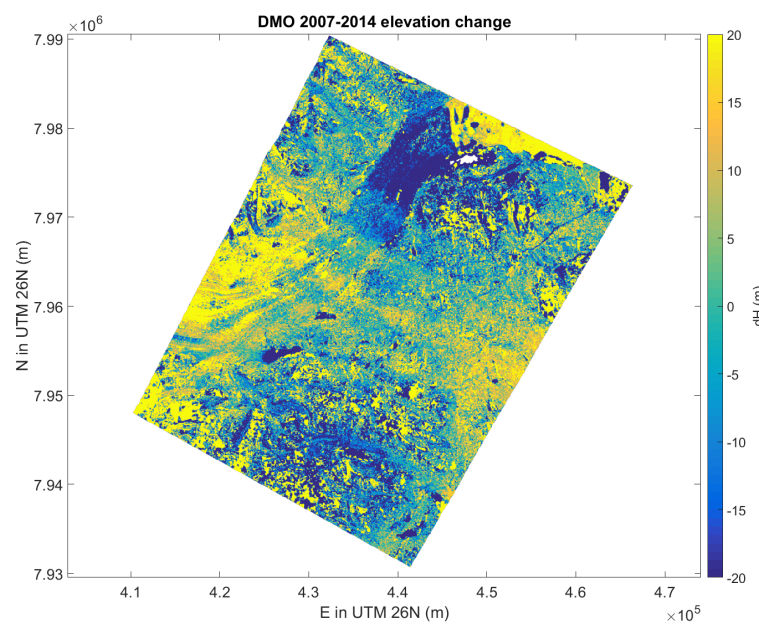

Figure 10: Measured elevation change from co-registered 2007 and 2014 DMO DEMs.

\section{IMPLEMENTATION}

The across-track jitter estimations and corrections, as well as the DEM and orthoimage production, were implemented in the free open source MicMac photogrammetric library (developed at the French National Institute of Geographic and Forest Information IGN - (Pierrot-Deseilligny et al., 2016) and (Pierrot-Deseilligny, 2016)). A ready to use software to produce DEMs and orthoimages from Level 1A ASTER products is therefore available.

However, our implementation uses the MatLab HDF tools to extract the data from the files provided by NASA as two destriped tiff images and two xml files containing the positions of the satellites and the lattice points in J2000.00 ECEF Cartesian coordinates as well as the lattice points in image coordinates. The postprocessing of the DEMs into elevation change products is also currently implemented in MatLab. Future development will port these capabilities into the MicMac library as well.

\section{CONCLUSION}

In continuation to the work presented in (Girod et al., 2015) on the production of improved DEMs from ASTER L1A products with focus on removal of effects from cross-track jitter, we have now extended the method to also deal with the remaining effect from the along-track jitter component and have shown that it is possible to fit corrections to the bias observed on the stable ground and produce MMASTER dDEMs that are suitable to survey glacier volume change, while it proved challenging to obtain good data with the DMO products.

When comparing two scenes of the same area to analyze elevation change in the terrain, MMASTER products show good accordance with SPIRIT products (that have themselves been proven to be adequate for mapping glacier elevation change by (Korona et al., 2009)). We can therefore conclude that, when sufficient stable ground is present for the fitting of corrections, the MMASTER products can be used for mapping glacier elevation change with an accuracy better than $\pm 10 m$, given that sufficient visual contrast is available in the data to permit matching of stereoparallaxes. This possibility opens a range of new perspectives for glacier change detection in view of the large and 15-year long global-scale archive of ASTER stereo data, its recent free availability, and the fact that the ASTER stereo sensor is still operational and will continue to acquire new data in the foreseeable future.

\section{ACKNOWLEDGMENTS}

This study was funded by the European Research Council under the European Union's Seventh Framework Programme (FP/20072013)/ERC grant agreement no.320816 and by the ESA project Glaciers_cci (4000109873/14/I-NB). Acquisition of the ASTER images was guided by NASA JPL, through the ASTER science team and the Global Land Ice Measurements from Space (GLIMS) initiative. The SPOT5-HRS DEMs were obtained through the IPY-SPIRIT program ((Korona et al., 2009)) (C)CNES and SPOT Image 2007 and 2014. We also acknowledge the help from Marc Pierrot-Deseilligny and the MicMac team at IGN/ENSG for their help in the development of the software.

\section{REFERENCES}

Baudoin, A., Schroeder, M., Valorge, C., Bernard, M. and Rudowski, V., 2004. The hrs-sap initiative: a scientific assessment of the high resolution stereoscopic instrument on board of spot-5 by isprs investigators. International Archives of Photogrammetry, Remote Sensing and Spatial Information Sciences 35(B1), pp. 372-378.

Girod, L., Nuth, C. and Kääb, A., 2015. Improvement of dem generation from aster images using satellite jitter estimation and open source implementation. ISPRS - International Archives of the Photogrammetry, Remote Sensing and Spatial Information Sciences XL-1-W5, pp. 249-253.

Kääb, A., Lamare, M. and Abrams, M., 2013. River ice flux and water velocities along a $600 \mathrm{~km}$-long reach of lena river, siberia, from satellite stereo. Hydrology and Earth System Sciences 17(11), pp. 4671-4683. 
Korona, J., Berthier, E., Bernard, M., Rémy, F. and Thouvenot, E., 2009. Spirit. spot 5 stereoscopic survey of polar ice: reference images and topographies during the fourth international polar year (2007-2009). ISPRS Journal of Photogrammetry and Remote Sensing 64(2), pp. 204-212.

Nuth, C. and Kääb, A., 2011. Co-registration and bias corrections of satellite elevation data sets for quantifying glacier thickness change. The Cryosphere 5(1), pp. 271-290.

Pierrot-Deseilligny, M., 2016. Micmac webpage.

Pierrot-Deseilligny, M., Rupnik, E., Girod, L., Belvaux, J., Maillet, G., , Deveau, M. and Choqueux, G., 2016. MicMac, Apero, Pastis and Other Beverages in a Nutshell! ENSG, IGN, ChampsSur-Marne, France.

SILC, 2015. Sensor information laboratory corp software's silcast ver.2.00 webpage.

Wang, D. and Kääb, A., 2015. Modeling glacier elevation change from dem time series. Remote Sensing 7(8), pp. 10117-10142.

Winsvold, S. H., Kääb, A. and Nuth, C., 2016. Regional glacier mapping using optical satellite data time series. IEEE Journal of Selected Topics in Applied Earth Observations and Remote Sensing PP(99), pp. 1-14. 